\title{
Schnittstellenfragen erörtern und klären
}

\author{
Bruno Kesseli \\ Dr. med. et lic. phil., Chefredaktor
}

Auch in der Ausgabe 2016 bot die traditionelle Journée de réflexion des Schweizerischen Instituts für ärztliche Weiter- und Fortbildung (SIWF) und des Kollegiums der Dekane der Medizinischen Fakultäten der Schweiz (Collège des Doyens) eine anregende Mischung aus Informationen, Ideen und Diskussionsbeiträgen rund um die ärztliche Bildung.

«Es sind praktisch alle da», konnte Werner Bauer, als Präsident des SIWF einer der Gastgeber der Journée de réflexion 2016, in seiner Begrüssungsansprache konstatieren. Damit lag er zweifellos richtig, denn es war in der Tat eindrücklich, wie viele in die ärztliche Bildung involvierte Institutionen und Organisationen mit namhaften Repräsentanten an der Tagung vertreten waren. Entsprechend dem Namen der Veranstaltung solle der Reflexion, dem Gedankenaustausch und der Diskussion viel Raum gegeben werden, hielt Werner Bauer fest. Insbesondere biete der Anlass einen idealen Rahmen, um «Schnittstellenfragen» zu erörtern und zu klären. Der SIWF-Präsident deutete bereits an, was im Verlauf der Tagung immer wieder zur Sprache kam: Aus-, Weiter- und Fortbildung sollen nicht in sich geschlossene Blöcke sein, sondern als Kontinuum konzipiert werden. Dieses soll dadurch charakterisiert sein, dass auf jeder Stufe Kompetenzen erworben werden, auf denen die folgende aufbauen kann.

Die «Journée» fand diesmal nicht wie in den letzten Jahren üblich in Brunnen, sondern in Luzern statt, was die Anreise für die Westschweizer etwas verkürzte, wie Henri Bounameaux mit einem Augenzwinkern vermerkte. Der Dekan der medizinischen Fakultät der Universität Genf und aktuelle Präsident des Collège des Doyens amtete als Co-Gastgeber und -Moderator der Veranstaltung.

\section{Auf den «SCLO» folgen die «PROFILES»}

Der erste Themenblock stand unter dem Titel «Die Phasen ärztlicher Bildung und ihre zukünftige Entwicklung». Er wurde eröffnet durch Pierre-André Michaud

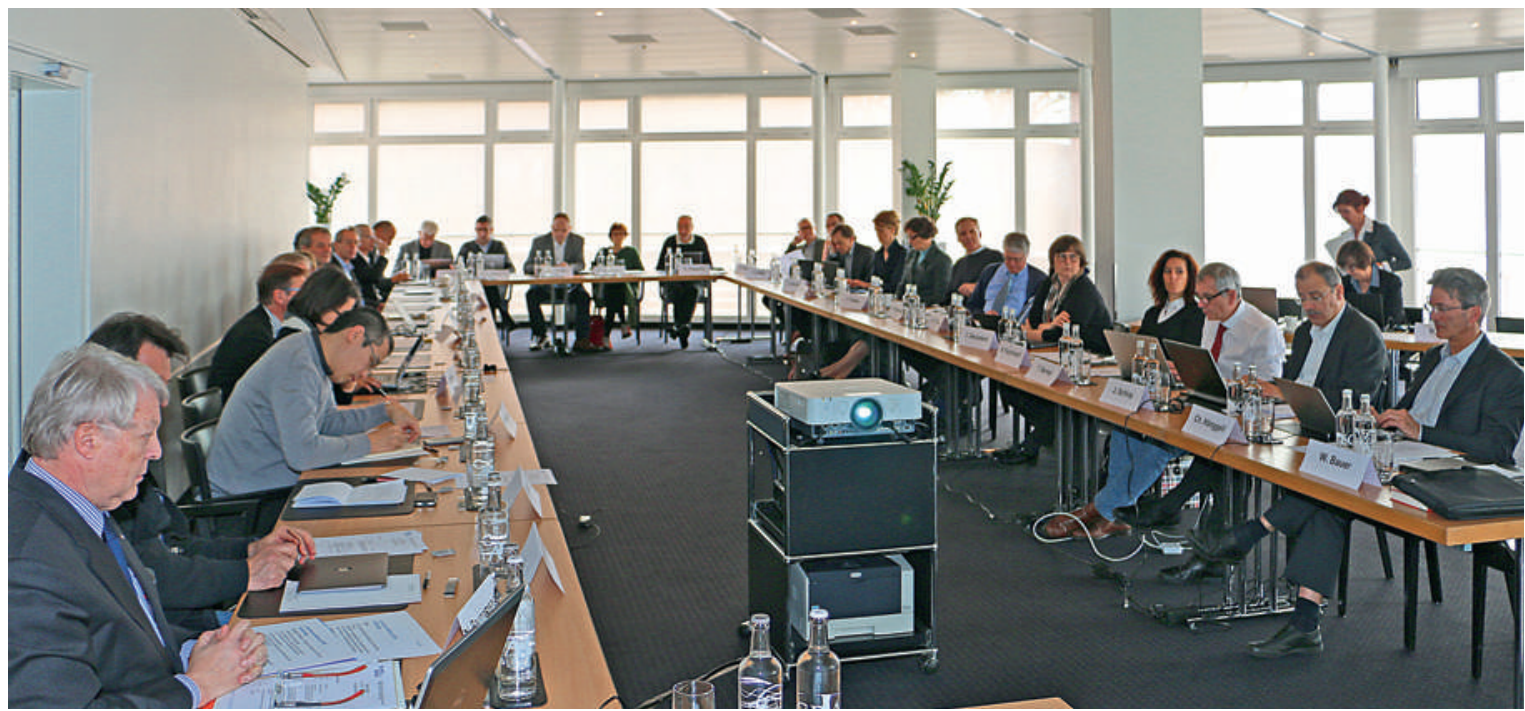

Ihrem Namen entsprechend bietet die «Journée de réflexion» Expertinnen und Experten aus dem Bereich der ärztlichen Bildung Raum für Gedankenaustausch und Diskussion. 


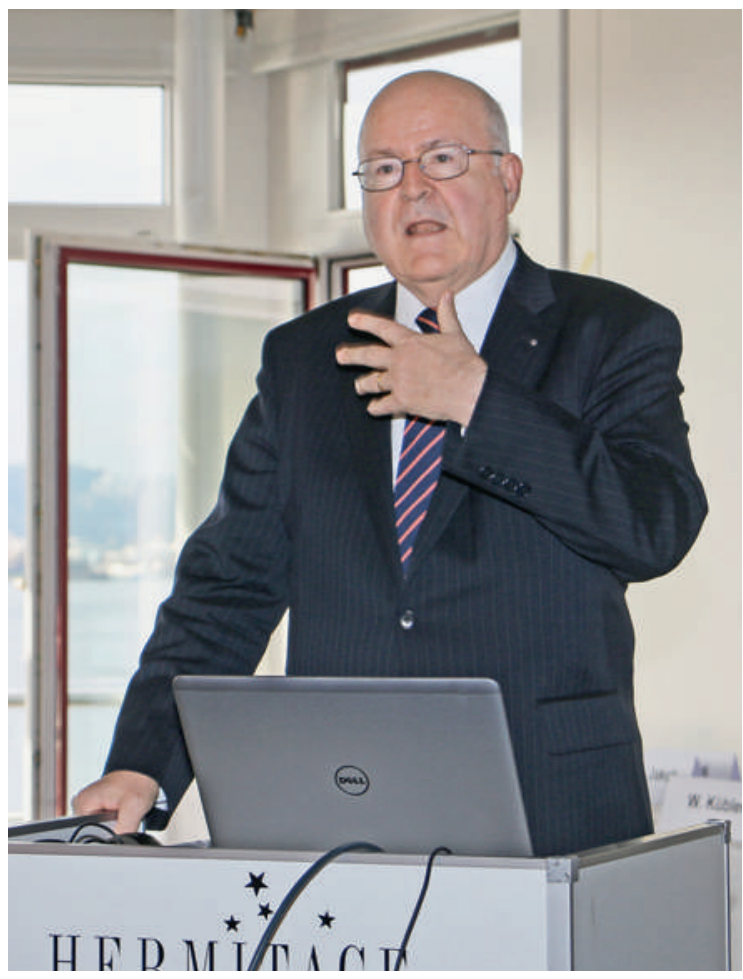

"Alle da»: SIWF-Präsident Werner Bauer eröffnete die Tagung.

von der Schweizerischen Medizinischen Interfakultätskommission (SMIFK), der den neuen Lernzielkata$\log$ PROFILES vorstellte. Dieses Akronym steht für «Principal Relevant Objectives and Framework for Integrated Learning and Education in Switzerland». Die PROFILES lösen den SCLO ab, den «Swiss Catalogue of Learning Objectives» für Medizinstudenten. Sie stehen für einen kompetenzbasierten Ansatz, der die Flexibilität und Autonomie der Studierenden sowie deren «Clincal reasoning» verbessern soll. Ziel ist es, mittels PROFILES Ärztinnen und Ärzte auszubilden, die nach Studienabschluss «ab dem ersten Tag ihrer Assistenzarztzeit in den Weiterbildungsstätten einsatzbereit sind». Eine Schlüsselrolle in diesem Konzept spielen die EPA, die «Entrustable Professional Activities». Darunter werden berufliche Fähigkeiten verstanden, die so sicher beherrscht werden, dass sie von der betreffenden Fachperson ohne Supervision ausgeübt werden können. EPA basieren auf der Integration verschiedener Kompetenzen und Disziplinen und sollen bereits im Studium nach einem Stufenkonzept erworben werden. Schon beim Antreten ihrer ersten Stelle müssten Assistenzärztinnen und -ärzte auf dieser Basis in der Lage sein, beispielsweise Notfälle während der ersten 20-30 Minuten fachlich korrekt zu managen.

Obschon das Konzept der PROFILES wohl allen Anwesenden grundsätzlich bekannt war, schien das Referat von Pierre-André Michaud einen Neuigkeitswert zu haben, löste es doch eine angeregte Diskussion zu verschiedenen Fragen aus. Peter Meier-Abt, Präsident der Schweizerischen Akademie der Medizinischen Wissenschaft (SAMW), interessierte sich dafür, ob die Studierenden unter dem neuen Regime auch lernten, was $\mathrm{EBM}^{*}$ sei, ob sie etwas über «Big Data», über personalisierte Medizin und weitere Themen von zunehmender Bedeutung erfahren würden. Reto Meuli, Direktor der Ecole de formation postgraduée der medizinischen Fakultät Lausanne, fragte nach, ob der Erwerb von Kompetenzen in Bereichen wie Ethik und Gesundheitspolitik abgedeckt sei. Pierre-André Michaud konnte in beiden Fällen bestätigen. Er wies auch darauf hin, dass die Installation einer interaktiven Plattform vorgesehen sei, die Anpassungen von PROFILES ermöglichen würde. Es folgen nun eine Vernehmlassung bei den Fakultäten, eine Überarbeitung unter Einbezug verschiedener Gremien und schliesslich die Approbation durch die SMIFK "unter den Augen des BAG», das ebenfalls involviert sei, wie Michaud festhielt. Der Beginn der Implementierung von PROFILES ist für 2017 vorgesehen.

\section{Weiterbildung in Zeiten des Struktur- wandels}

Werner Kübler, Direktor des Universitätsspitals Basel, illustrierte in seinem Referat zum Thema «Spannungsfeld Spitalbetrieb und ärztliche Bildung» an zahlreichen Beispielen, wie der Strukturwandel im Spitalbereich sich auf die ärztliche Weiterbildung auswirkt. Die Spitäler sind mit etlichen Herausforderungen öko-

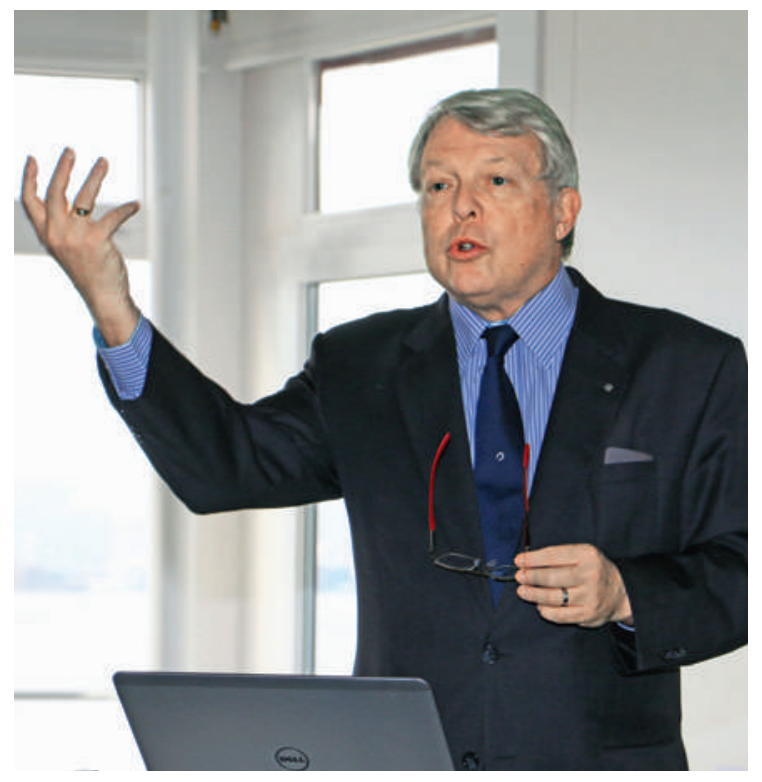

Co-Gastgeber und -Moderator: Henri Bounameaux, Präsident des Collège des Doyens. 


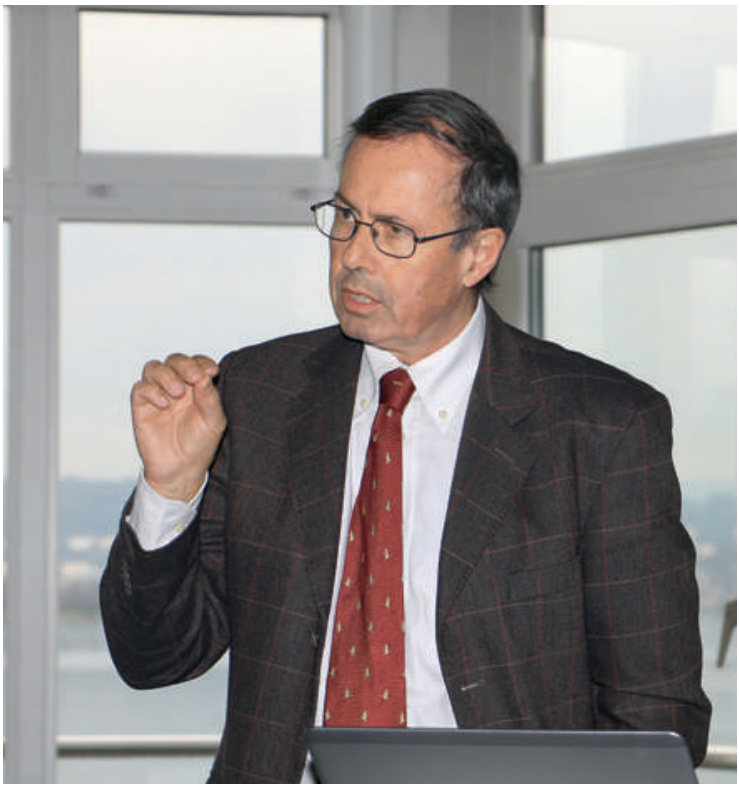

Pierre-André Michaud stellte den neuen Lernzielkatalog «PROFILES» vor.

nomischer und organisatorischer Art konfrontiert, die mit den bisherigen Weiterbildungsmodellen nicht immer kompatibel sind. Kübler nannte in diesem Zusammenhang etwa die Bildung interdisziplinärer Zentren, multiple Kooperation mit «wandernden Ärzten» oder Entwicklungen weg von Fachkliniken hin zu interprofessionellen Prozessteams im Zuge einer patientenorientierten Medizin. Diese Entwicklungen stehen teilweise in Konflikt mit Struktur- und Organisationsvorgaben für die Anerkennung von Weiterbildungsstätten. Es entstünden, so Kübler, «labile Gebilde». Allerdings böten diese Entwicklungen auch Chancen. Er nannte als «uneingeschränkt positives Beispiel» das Netzwerk Weiterbildung Hausarztmedizin Nordwestschweiz. Aus seiner Sicht zeichnen sich in Bezug auf die Weiterbildung Gewichtsverschiebungen $a b$, beispielsweise von der Weiterbildungsstätte als Struktur zum Weiterbildungsinhalt beziehungsweise von der Weiterbildungsstätte als Ort zum Weiterbildungsnetzwerk. Der Stellenwert interdisziplinärer medizinischer Fähigkeiten werde vermutlich zunehmen, die vorwiegend inhaltlich ausgerichtete Fachweiterbildung dürfte sich in Richtung der Bildung einer «professionellen Persönlichkeit» entwickeln. Die Diskussion bestätigte die Notwendigkeit, neuen Schnittstellenproblemen und Strukturen mit flexiblen, modularen, auf Vernetzung aufbauenden Lösungen gerecht zu werden. Aus Sicht des SIWF ist dies auf der Basis eines frühzeitigen Dialogs zwischen den involvierten Partnern durchaus möglich.

\section{«Willkommen in der Schweiz»}

Dass man als Arzt auch in der Politik sehr erfolgreich sein kann, beweist der Tessiner Nationalrat und FDPFraktionschef Ignazio Cassis. An der «Journée de réflexion» stand er seinem Gesprächspartner Werner Bauer und dem Publikum in einem angeregten «Gedankenaustausch» Rede und Antwort. Die Vorstellung, als Arzt sei man «automatisch» auch ein Lobbyist der Ärzteschaft, ist für ihn ein "romantischer Gedanke». Er fühle sich vielmehr als überzeugter Lobbyist für die liberalen Werte. Was dies bedeutet, äusserte er für einen Politiker erstaunlich frei von der Leber weg. Der Hinweis auf das Fehlen einer nationalen Gesundheitspolitik entlockte ihm den trockenen Kommentar «Willkommen in der Schweiz!». Es sei eben genau ein Charakteristikum unseres föderal organisierten Landes, dass es nur eine schwache nationale Gesundheitspolitik gebe. Auf die Frage, wie man die "Anarchie» im Gesundheitswesen etwas regeln könne, meinte er, unser System sei nicht anarchisch, sondern im Gegenteil hyperreguliert. Die FDP-Lösung wäre ein regulierter Markt, in dem die Tarifpartner Preise aushandeln würden. Bis anhin sei dies nicht oder nur sehr beschränkt möglich. Durch Bürokratie und Überregulierung hätten wir uns viele Probleme geschaffen. Insbesondere die vielen Verordnungen durch die Verwaltung wirkten lähmend. Dass man den Schwarzen Peter nicht generell der Verwaltung zuschieben kann, räumte Cassis auf eine Intervention von Nadine Facchinetti allerdings ein. Die Leiterin ad interim der Abteilung Gesundheitsberufe des Bundesamts für

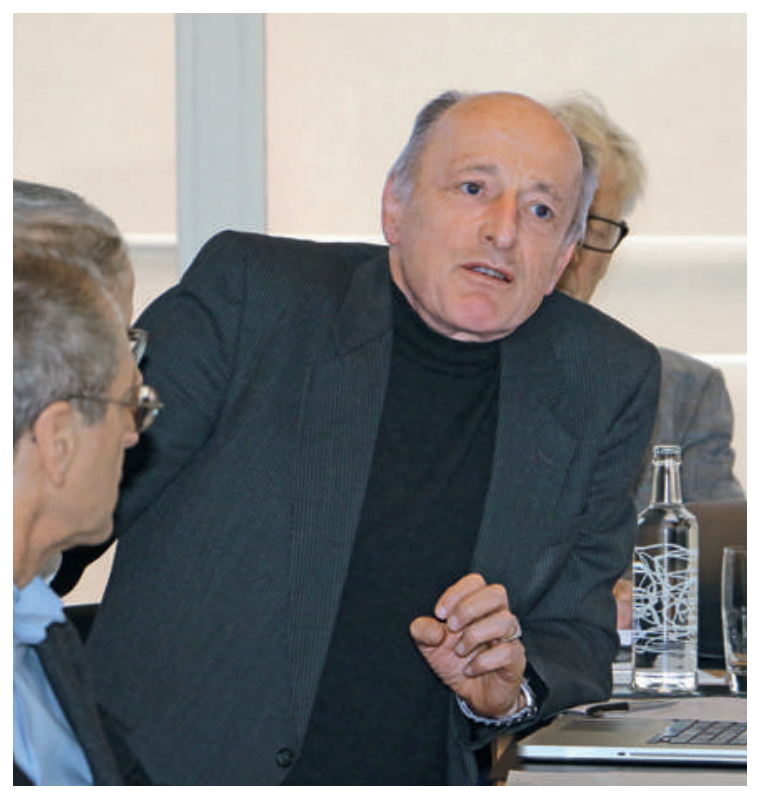

«EBM», «Big Data» und «personalisierte Medizin»: Peter Meier-Abt, Präsident der SAMW. 


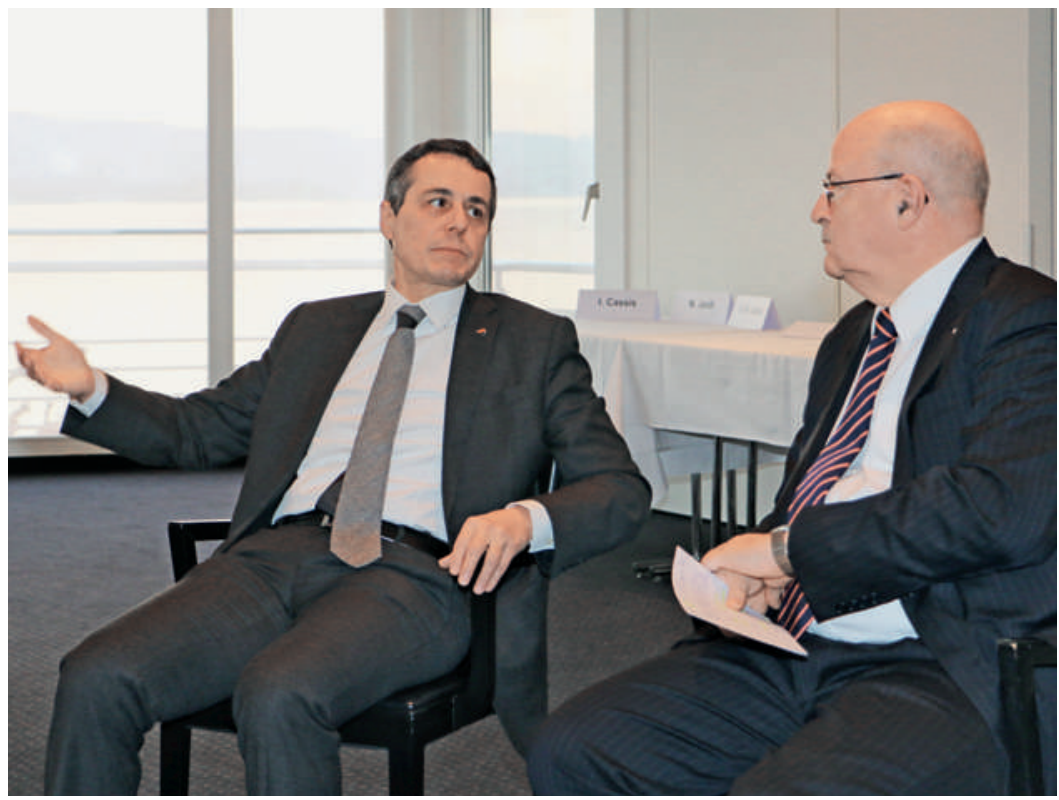

Lobbyist für die liberalen Werte: Ignazio Cassis (links) im Gespräch mit Werner Bauer.

Gesundheit hatte darauf hingewiesen, dass es beim Medizinalberufegesetz MedBG das Parlament gewesen sei, das eine schlanke Verordnung aufgebläht habe.

\section{Newsflash mit breitem Spektrum}

Ein Newsflash mit aktuellen Mitteilungen aus dem SIWF und dem Collège des Doyens eröffnete den zweiten Tag der Veranstaltung. Informiert wurde über Themen wie die Akkreditierung 2018 der Weiterbildungsgänge durch das BAG, die Plattform «Ärztliche Bildung» von GDK und BAG sowie verschiedene Projekte des SIWF zur Förderung und Unterstützung der Weiterbildung. Christoph Hänggeli, Geschäftsführer des SIWF, lieferte kurze Updates zum komplexen e-Logbuch-Projekt, zur Fortbildungsplattform sowie zur Informatisierung im Bereich der Weiterbildungsstätten. Auch die Anzahl erteilter Facharzttitel, die Revision des MedBG und die korrekte Ausschreibung von Titeln waren Gegenstand seiner Ausführungen [1].

\section{Eine Lanze für das Wissen}

Josef Pfeilschifter begann sein mit Leidenschaft vorgetragenes Referat ganz am Anfang, nämlich bei Adam und Eva, der Unterscheidung von Gut und Böse und der Vertreibung aus dem Paradies. Der paradiesische Zustand des Menschen, so der Dekan des Fachbereichs Medizin der Goethe-Universität Frankfurt am Main, lasse sich mit «kompetent, aber unwissend» umschreiben. Seither sei der Mensch «kompetent, aber wissend». Damit war das Terrain vorbereitet für den ersten einer ganzen Reihe von rhetorischen Paukenschlägen: «Den paradiesischen, altsteinzeitlichen Zustand - kompetent, aber unwissend - wieder herzustellen ist seit einiger Zeit Ziel der Universitätspolitik in Deutschland.»

Dass Pfeilschifter nicht weiss, wovon er spricht, kann man ihm kaum vorwerfen. Er war Mitglied der Kommission, die den "Nationalen Kompetenzbasierten Lernzielkatalog Medizin» (NKLM) erarbeitet hat. Allerdings sei es ihm dort primär darum gegangen, «Ärgeres zu verhindern" und "die Rolle der Wissenschaft und des Wissens in der Ausbildung hochzuhalten». Der auch in Deutschland im Trend liegenden Kompetenzorientierung begegnet er mit höchster Skepsis. Die Kompetenz, über Wissen verfügen zu können, genüge nicht: «Jemand, der lesen lernen könnte, es aber nicht kann, bleibt ein Analphabet.» Wissensbesitz sei die Voraussetzung für Wissenserwerb. Auch Fertigkeiten seien eine Form von Wissen, nämlich prozedurales Wissen.

Pfeilschifter brach eine Lanze für das sogenannte «kristalline Wissen» von Daten und Fakten, das unerlässlich sei, da es erst die Bausteine liefere für die kreative Assoziation, ja für die Kognition selbst. Ein "Wissenskanon" sei ein wesentliches integratives Moment in jedem Gemeinwesen. Diesen gemein samen soziokulturellen Bezugsrahmen drohten wir zu verlieren. Möglicherweise sei genau das gewollt. Wir «bereiten den Boden für Rattenfänger, Kreuzzügler, Dschihadisten, Homöopathen und Gesund- und Krankbeter aller Couleurs, die das Wissensvakuum nur allzu

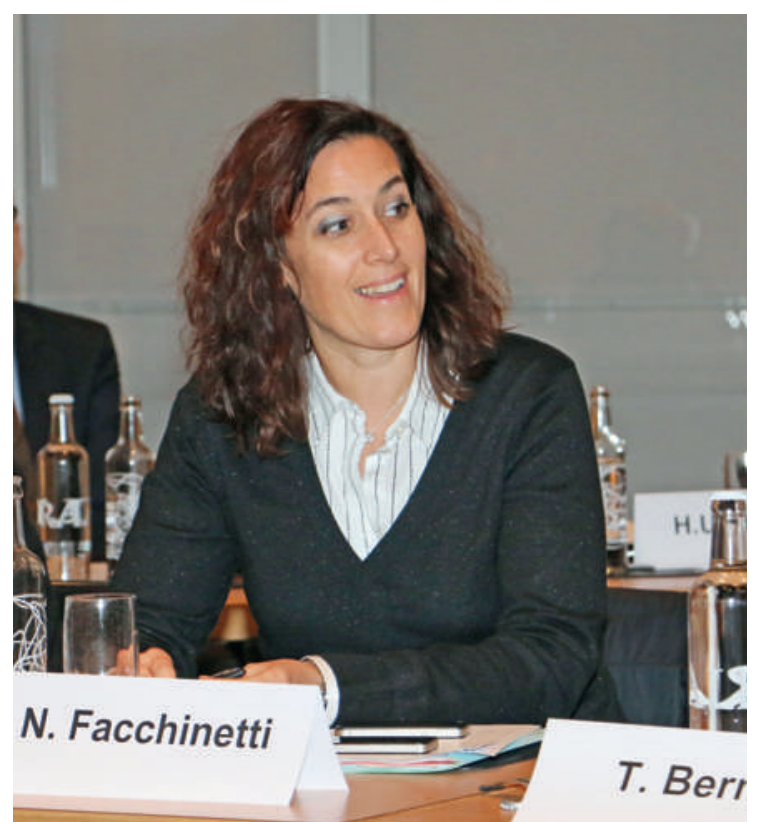

Ortet bürokratische Tendenzen auch im Parlament: BAG-Vertreterin Nadine Facchinetti. 


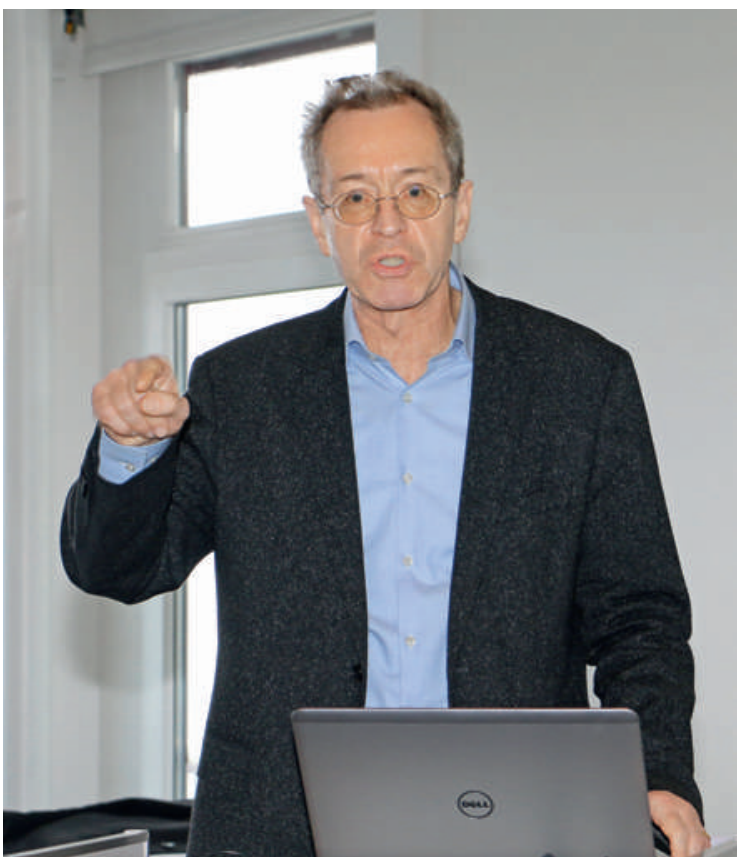

Plädoyer für Wissen und Wissenschaft: Josef Pfeilschifter.

gerne mit ihrem normativen Geschwätz füllen». Mittlerweile werde tatsächlich gefordert, an den Universitäten "safe spaces des Unwissens» einzurichten. "Wissen stört", so Pfeilschifter, und "wer bestimmen kann, welches Wissen stört, hat die Macht.»

Insbesondere in der Wissenschaft sei eine "Outputorientierte Problemlösungskompetenzdidaktik» fatal, da Wissenschaft prinzipiell ein ergebnisoffener Prozess sei. Wissenschaft zu betreiben sei der Job jedes Universitätsdozenten. «Wir verrieten uns selbst, wenn wir sie unseren Studierenden vorenthielten.» Und, last but not least, könne sie auch Spass machen, die Wissenschaft.

\section{Offene Fragen der ärztlichen Bildung}

(1847-1919), kanadischer Mediziner und Humanis mit grossem Einfluss auf die Entwicklung der medizinischen Weiterbildung.

bkesseli[at]emh.ch
Nachdem Henri Bounameaux in seiner Funktion als Präsident eine Stellungnahme des Collège des Doyens zur ärztlichen Ausbildung in der Schweiz vorgestellt hatte [2], befasste sich der zweite Themenblock mit offenen Fragen der ärztlichen Bildung. Davon gibt es eine ganze Reihe. Dass einmal mehr die Finanzierung der Weiterbildung angesprochen wurde, überraschte nicht. Werner Bauer nutzte die Gelegenheit, dem Zentralsekretär der Gesundheitsdirektorenkonferenz die Frage zu stellen, ob das SIWF zur Lösung des Problems beitragen könne. Der angesprochene Michael Jordi empfahl, via die Kantonalen Ärztegesellschaften an die jeweiligen Gesundheitsdepartemente zu gelangen. Insbesondere in Kantonen, die «Sparübungen» machten, könnten solche Interventionen etwas bewirken.

Zur Sprache kamen auch die Optimierung der Zusammenarbeit zwischen den Fakultäten, den Universitätsspitälern und dem SIWF, die Definition von Lernzielen und eine mögliche Regulierung der Weiterbildungsgänge in der Schweiz. Zum letzten Punkt durften einige der Anwesenden ihre «To-do-List» ergänzen: Die Fakultäten und die SMIFK sollten prüfen, ob und wie die Voraussetzungen für den Erwerb eines Doktortitels vereinheitlicht werden könnten. Dem SIWF obliegt die Klärung der Frage, ob sich die Anzahl der Publikationen reduzieren lässt, die für den Erwerb eines Facharzttitels nötig ist.

Zum Schluss kam wieder einmal Sir William Osler** zum Zug. Seine Botschaft dürfte die Gemüter derer aufgehellt haben, die angesichts der ungelösten Probleme um die Qualität der ärztlichen Bildung fürchten. Oslers folgende, 1909 im JAMA erschienene Aussage gibt im Gegenteil zu Optimismus Anlass: «A faculty without its troubles is always in a bad way - the water should be stirred. Some ferment should be brewing; the young men should always be asking for improvements, to which the old men would object.»

\section{Bildnachweis}

Fotos Bruno Kesseli

\section{Literatur}

1 Weitergehende Informationen zu den genannten Themen finden sich in folgenden SÄZ-Artikeln:

Kesseli B. Auf vielen Feldern aktiv. Plenarversammlung des SIWF am 26. November 2015 in Bern. Schweiz Ärztezeitung. 2016;97(4):124-8.

Hänggeli C, Kuhn HP, Linder B. Titelausschreibung leicht gemacht. Schweiz Ärztezeitung. 2015;96(50-51):1834-6.

2 Dieses Dokument ist mittlerweile in der SÄZ publiziert worden: Bounameaux H, Eggli P, Gasser T, Tissot JD, Weber R. Statement regarding medical education in Switzerland. Schweiz Ärztezeitung. 2016;97(12-13):461-2. 